\title{
Does mental rotation emulate motor processes? An electrophysiological study of objects and body parts
}

\author{
Marta Menéndez Granda \\ University of Geneva \\ Giannina lannotti \\ University of Geneva \\ Alexandra Darqué \\ University of Geneva \\ Radek Ptak ( $\nabla$ radek.ptak@unige.ch ) \\ University of Geneva
}

\section{Research Article}

Keywords: mental rotation, ERP, embodiment, simulation, angular gyrus, parietal lobe

Posted Date: February 22nd, 2021

DOI: https://doi.org/10.21203/rs.3.rs-155317/v1

License: (9) This work is licensed under a Creative Commons Attribution 4.0 International License.

Read Full License 


\section{Does mental rotation emulate motor processes? An electrophysiological study of objects and body parts}

Abbreviated title: Motor emulation in mental rotation

Marta Menéndez Granda ${ }^{*}$, Giannina Rita lannotti ${ }^{1,3^{*}}$, Alexandra Darqué ${ }^{1}$ and Radek Ptak ${ }^{1,2}$

${ }^{1}$ Laboratory of Cognitive Neurorehabilitation, Department of Clinical Neurosciences, Faculty of Medicine, University of Geneva, 1206 Geneva, Switzerland

${ }^{2}$ Division of Neurorehabilitation, University Hospitals of Geneva, 1206 Geneva, Switzerland

${ }^{3}$ Swiss Foundation for Innovation and Training in Surgery, University Hospitals of Geneva, 1206 Geneva, Switzerland

*Shared first authorship

\section{Correspondence:}

Radek Ptak, PhD

radek.ptak@unige.ch

Division of Neurorehabilitation

Department of Clinical Neurosciences

Geneva University Hospital

26, Av. de Beau-Séjour

1211 Geneva 14

Switzerland

Tel.: +41-22/372 3524

Conflict of interest: The authors declare no conflict of interest. 


\begin{abstract}
Several arguments suggest that mental rotation (MR) and motor planning may share embodied neural mechanisms, but the overlap between cognitive processes recruited during MR of objects and body parts is not well established. We here used high-density EEG to examine the cognitive similarity between MR of nonmanipulable objects (chairs) and bodily stimuli (hands). We selected chairs because they may appear in a recognizable left-right orientation and are not automatically associated with a manual action. Participants had identical response options for both types of stimuli, and they gave responses orally in order to prevent possible interference with motor imagery. MR of hands and chairs generated very similar behavioral responses, timecourses and neural sources of evoked-response potentials (ERPs). ERP segmentation analysis revealed distinct time windows during which differential effects of stimulus type and angular disparity were observed. An early period (90-160 ms) differentiated only between stimulus types, and was associated with occipito-temporal activity. A later period (290-330 ms) revealed strong effects of angular disparity, associated with electrical sources in the right angular gyrus and primary motor/somatosensory cortex. These data suggest that spatial transformation processes and motor planning are recruited simultaneously, supporting the involvement of motor emulation processes in MR.
\end{abstract}

Keywords: mental rotation; ERP; embodiment; simulation; angular gyrus; parietal lobe 


\section{Introduction}

The evolution and use of higher cognitive functions are submitted to strong biological constraints, which provide an upper bound on complexity, interdependence and energy consumption. Increasing evidence shows that the human brain deals with these constraints by reutilizing basic motor and sensory resources for higher cognitive processing ${ }^{1-5}$. While an intense debate exists regarding the degree to which symbolic knowledge is embodied ${ }^{6,7}$, even critics agree that some form of embodiment must underlie mental rotation MR; ${ }^{8}$. This conclusion is based on several arguments. First, a well-established linear relationship exists between reaction time and degree of the rotation stimulus, expressed as angular disparity from a zero-degree (upright) reference item ${ }^{9,10}$. This pattern is what would be expected from a process that emulates the temporal characteristics of a physical rotation, for example when subjects create a mental image and then manipulate it until a match with the probe is found. Second, MR capacity seems to run parallel to motor development, as motor control is an independent predictor of MR performance in young children ${ }^{11}$. Third, functional neuroimaging studies show that MR activates regions of posterior parietal and premotor cortex similar to those involved in motor planning, suggesting strongly overlapping neural substrates ${ }^{12-15}$.

Though these observations suggest that MR and motor planning may share embodied neural mechanisms, the degree of overlap between cognitive processes involved in MR of objects and body parts is not well established ${ }^{16}$. Participants performing MR of objects often describe a visual imagery strategy: they create an image of the stimulus and then mentally rotate it until it matches the original ${ }^{17}$. In contrast, MR of body parts appears to require some form of motor imagery ${ }^{18,19}$. For example, when participants are asked to judge the laterality of a shown hand, they mentally simulate moving their own hand until it reaches the orientation of the hand pictured ${ }^{20,21}$. Functional imaging studies comparing MR of objects and body parts support the involvement of distinct, yet partially overlapping cognitive processes by showing similar neural activations. Several MR studies of objects revealed bilateral activations of posterior parietal cortex (PPC), while MR of hands more often activated motor and premotor areas ${ }^{22-28}$. Meta-analyses support the involvement of posterior parietal and motor/premotor regions in MR, but they do not distinguish between different types of stimuli ${ }^{12,15,29}$. A more recent meta-analysis examined differences between activations related to MR of bodily and non-bodily stimuli and found that the former activated frontoparietal areas including lateral premotor and supplementary motor cortices while the latter generated right superior and inferior parietal activations ${ }^{16}$. However, few of the included studies compared the two types of stimuli directly and with the same subjects. Vingerhoets et al. ${ }^{30}$ compared MR of tools and body parts and found premotor activations when subjects performed MR of hands and tools, although the former showed greater activity in right precentral cortex. While there is some evidence that MR of objects and body parts both rely on similar neural substrates and motor emulation processes, at least three important limitations remain. First, manipulable objects (such as tools) might activate motor areas simply because they are automatically associated with hand use. Second, activations of motor/premotor areas to object rotation might at least partly be linked to the fact that subjects answered with a finger press. Finally, even if fMRI studies show common activations for objects and body parts, they cannot resolve the temporal order of brain regions activated in quick succession.

We here took advantage of the excellent temporal resolution of the electroencephalogram (EEG) to analyze different processing stages involved in MR of objects and hands. Modern high-resolution EEG allows identification of electrical current sources with improved precision, thus providing an acceptable trade-off for the study of physiological processes varying in space and time. Previous event-related potential (ERP) studies seem to agree on the distinction of two relevant time windows: an early phase $(\sim 170-200 \mathrm{~ms})$ that might be associated 
with visual classification or identification of the visual stimulus, followed by a later phase reflecting the mental transformation of the image in the mind $\sim 400-700 \mathrm{~ms} ;{ }^{31}$. In this later phase, several studies have observed more negative amplitudes of ERPs over the parietal cortex as a function of angular disparity ${ }^{32-35}$. However, there is a lack of ERP studies comparing MR of objects and body parts directly. The closest to answering our question whether MR of all types of stimuli relies on motor emulation comes a recent study by Jansen et al. ${ }^{35}$. These authors compared MR of geometric figures, whole human bodies and body postures, and identified an early period $\sim 200-400$ ms after stimulus onset, reflecting distinct activation patterns between abstract and embodied figures over parietal and central electrodes. In addition, a late period ( 400-600 ms) was characterized by discrete ERP patterns over central and frontal electrodes. However, this study emphasized differences, rather than pointing to similarities between MR of bodily stimuli and objects. For this reason, further comparisons are necessary to determine the precise degree of overlap between cognitive processes and underlying neural sources of activations.

The aim of the present study was to examine to what extent MR of non-manipulable objects (chairs) and bodily stimuli (hands) share cognitive processes, time courses and neural sources. We chose chairs because they are well-known stimuli, do not afford a specific hand action and can easily be distinguished as looking toward the left or the right. This was necessary because we wanted to create experimental conditions in which participants always gave the same response ('left', or 'right') irrespective of the type of stimulus. In addition, we asked subjects to respond orally instead of by using their hands/fingers. This differs from most previous studies, and was motivated by the expectation that any kind of manual action might interfere with motor imagery of hands and thus perturb motor emulation processes involved in MR.

\section{Results}

We showed hands/chairs at different angular disparities to healthy participants and asked them to indicate whether a depicted hand represented a left or right hand, or whether a chair was oriented leftward or rightward if put in its upright position (Figure 1a). We first present the behavioral results, followed by the ERP analyses.

\section{Behavioral results}

Figure $1 \mathrm{~b}$ shows for both stimulus types the increase of RTs as a function of angular disparity. The rANOVA with Stimulus type and Angular disparity as within-subjects factors yielded a statistically significant effect of Angular disparity on RTs $\left(F_{1.54,26.18}=106.33, p<0.001, \eta^{2}=0.862\right)$. Post-hoc comparisons revealed significant differences between all levels of angular disparity (all $p \leq 0.01$ ). The rANOVA also revealed a significant effect of Angular disparity on the percentage of errors $\left(F_{1.98,33.68}=4.97, p=0.013, \eta^{2}=0.226\right)$, which was due to a higher error rate for $120^{\circ}$ than $60^{\circ}(p=0.031)$ and $180^{\circ}$ vs. $0^{\circ}(p=0.029)$.

\section{Amplitude analysis}

Figure 2 shows the results of amplitude comparisons across the whole electrode set, as a function of Stimulus type, Angular disparity or the interaction of both factors. Non-parametric waveform analyses revealed statistically significant effects of Stimulus type between $\sim 160-190 \mathrm{~ms}$ and Angular disparity between $\sim 70-100 \mathrm{~ms}$ and $\sim 440$ - 
$500 \mathrm{~ms}$. In addition, the interaction between both factors yielded significant results between $\sim 120-150 \mathrm{~ms}, \sim 200$ $260 \mathrm{~ms}$ and $\sim 380-410 \mathrm{~ms}$. In order to examine whether these periods corresponded to EEG microstates we followed up with the ERP segmentation analysis.

\section{Results of ERP segmentation}

The meta-criterion for segmentation of the group-averaged ERPs associated with the four experimental conditions identified eight time segments (Figure 3a) and associated scalp EEG topographic maps (Figure $\mathbf{3 b}$ ). Group-averaged ERP permutation analysis across the two experimental factors (Stimulus type, Angular disparity) revealed significant results for Maps 1, 6, 7 and 8. Map 1 had an average length of 90-160 ms, corresponding to an early period of ERP differences, as reported in previous MR studies ${ }^{31}$. Maps 6-8 were located in a time window between $290-500 \mathrm{~ms}$, corresponding to a late period observed in previous studies ${ }^{32-35}$.

The statistical effects of each of the six map parameters are summarized in Table 1. Map 1 was only characterized by a main effect of Stimulus type on offset $(p=0.007$; Chairs $>$ Hands) and center of gravity $(p=$ 0.006; Chairs > Hands). Map 6 was distinguished by a significant interaction for duration $(p=0.004)$ and AUC ( $p$ $=0.001$ ) and was most specific for the condition Hand 180, where it was present between 290 and 360 ms. Map 7 exhibited strong main effects of Angular disparity as well as interaction effects for several parameters. It started around 310 ms for the condition Chair 180, was present for a longer period of time, ended later and had larger AUC for non-rotated than rotated stimuli (duration: $p=0.002$; offset: $p=0.004$; $A \cup C p=0.001$ ). In addition, the Angular disparity effect lasted longer for Chairs than Hands (offset, interaction effect: $p=0.047$ ), but had higher power for Hands than Chairs (interaction effect, AUC: $p=0.033$; mean GFP: $p=0.002$ ). Finally, it exhibited an earlier center of gravity for Chairs (interaction effect: $p=0.012$ ).

On the grand-averaged ERPs, Map 8 ( $430-500 \mathrm{~ms})$ was mainly present for rotated conditions. This map started earlier (onset: $p=0.0071$ ), lasted longer (duration: $p=0.018$ ) and was more activated (AUC: $p=0.021$ ) for chairs than hands. In addition, it exhibited main effects of Angular disparity for onset $(p=0.001)$, duration $(p=0.003)$ and AUC $(p=0.001)$, always in favor of rotated stimuli. Angular disparity effects were longer and stronger for Chairs than Hands (interaction, duration: $p=0.023$; AUC: $p=0.002$ ).

In sum, while Map 1 indexed differences of stimulus type Map 6 was specific for rotated hands, Map 7 reflected processing of upright as compared to rotated stimuli, and this especially for chairs. In contrast, Map 8 was mainly associated with stimulus rotation, again particularly for chairs.

\section{Map localization}

Since the duration of maps varied across conditions, we did not compute inverse solutions across the entire map intervals, but rather over time periods when each map was particularly strongly expressed (i.e., had high GFP). Figure 4 shows the inverse solution computed over the interval 120-150 ms that was associated with Map 1 in all four conditions. Since Map 1 was only characterized by a Stimulus effect, ERP sources were computed for chairs and hands irrespective of stimulus orientation. Both stimulus types were associated with bilateral activations of fusiform and posterior parahippocampal gyrus. For hands activations reached posteriorly into the left calcarine sulcus. Current sources for Map 6 were examined in the period 330-360 ms, independently for all 
four conditions. This map was associated mostly with right-hemisphere activations of the angular gyrus and medial parietal cortex (Figure 5). Importantly, there was also significant recruitment of the primary somatosensory cortex and primary motor cortex. The neural current sources were strikingly different for Map 7 (390-420 ms), which was essentially characterized by left mid- and anterior temporal activations (Figure 6). An exception was the condition Chair 180 , for which the inverse solution identified an area in the right medial and lateral temporal lobe. Finally, Map 8 (450-480 ms) was associated with left anterior temporal sources for Chair 0, right frontopolar sources for Chair 180 and bilateral dorsomedial sources for both hand conditions (Figure 7).

\section{Discussion}

Our findings show that MR of hands and chairs is characterized by very similar behavioral responses, timecourses and neural sources of ERPs, suggesting a strong overlap between underlying cognitive processes. Though some previous studies also compared MR of body parts and objects ${ }^{13,27,35,36}$, our MR paradigm distinguishes itself by three features. First, we used objects that are not associated with a distinctive manual response, and are therefore not expected to trigger actions implying the hands. Second, irrespective of stimulus type, subjects always had the same response options. Finally, in order to eliminate any interference of the response with motor imagery involving the hand participants were asked to respond orally. Given that the timecourse of ERPs is taken as predictor of the sequence of different cognitive processes recruited during a task it is critical for EEG studies to compare conditions that are equal regarding RT and error rate. The fact that the behavioral data of hands and chairs were indistinguishable regarding RTs and accuracy makes sure that differences of ERP patterns for hands and chairs cannot be attributed to differences in processing speed.

The segmentation analysis of group-averaged ERPs for upright and rotated stimuli revealed two time periods in which stimulus type and angular disparity had differential effects. An early period (between 90-160 ms, corresponding to Map 1) was characterized only by an effect of stimulus type. Previous EEG studies of visual processing have revealed that high-level perceptual analysis, such as rapid recognition of faces and other body parts, as well as identification of low-level object features occur within the initial $150 \mathrm{~ms}$ after stimulus presentation 37-39. Given the pure effect of stimulus type characterizing early processing, Map 1 therefore seems to be related to the early rapid identification of the stimulus pictured, regardless of its orientation. This interpretation is supported by the source localization analysis, showing bilateral activations in parahippocampal and fusiform gyri, brain regions that have been consistently implicated in the coding of distinct object categories (such as faces, objects or body parts) based on perceptual characteristics ${ }^{40-43}$.

Effects of angular disparity and an interaction between this factor and stimulus type appeared with Map 6, and thus considerably later than the pure stimulus effect. Several previous ERP studies have observed significant differences between upright and inverted stimuli in time periods starting at $\sim 400 \mathrm{~ms}$, an effect that has specifically been attributed to MR ${ }^{31,35}$. However, while these studies suggested a single cognitive component, our segmentation and source analyses support - akin to previous modelling studies ${ }^{44,45}$ - at least three distinct processing stages. The first stage (290-360 ms) was represented by Map 6 which was mainly present when rotated hands were manipulated. The source localization revealed an activation in the right inferior parietal cortex centered on the angular gyrus, which suggests that this segment might be related to the spatial transformation of the mental images. In fMRI studies the angular gyrus is activated when subjects are engaged in visuo-spatial 
judgments, such as the comparison of lengths or angles ${ }^{46-49}$. It has also been consistently identified as one of the most important brain regions supporting mental image transformations in MR ${ }^{12,16,29}$. We found angular gyrus activation irrespective of stimulus type, and only in the right hemisphere, which is compatible with a high-level, stimulus-independent process that depends strongly on the right hemisphere's superior capacity for visuo-spatial transformations ${ }^{50}$.

The presence of Map 7 suggests a distinct cognitive component, since it occurred significantly later than Map 6, and the interaction between stimulus type and angular disparity had a different effect on its presence. While Map 6 was particularly sensitive to the presence of rotated hands, the cognitive processes associated with Map 7 are more difficult to discern. On the one hand, this map started earlier for rotated chairs than all other conditions. On the other hand, several other aspects such as duration, offset and AUC differentiated this map between upright and rotated stimuli. This map also had distinct current sources, with right temporal activations for rotated chairs, and left temporal activations for all other conditions. Though it is difficult to identify a specific cognitive process, the temporal activations and time-course of Map 7 suggest that this stage might be relevant for semantic classification of rotated and upright stimuli. In accordance with this, Schendan and Maher ${ }^{51}$ showed that after early and rapid visuo-perceptual processing of the stimulus, visual and semantic encoding continues and eventually temporally overlaps with later-occurring ERP patterns associated with MR.

Map 8 indicates the involvement of a third cognitive sub-process, which is mainly present for rotated conditions, irrespective of stimulus type. Source localization analyses revealed principally right prefrontal activations, reaching from the right frontal pole medially toward the anterior cingulate gyrus. This anterior localization, together with the relatively late occurrence of Map 8, is compatible with decision processes related to response selection 52. This interpretation is supported by previous studies which have similarly identified late electrophysiological components occurring after the spatial transformations during MR ${ }^{53,54}$.

The main question motivating this study was whether MR of objects is grounded within motor emulation processes that mimic actual actions. An answer to this question rests on two main assumptions: first, that MR of hands activates motor processes similar to those activated during real hand actions; second, that MR of objects is sufficiently similar to MR of hands to trigger these same motor processes. Regarding the first assumption, functional imaging studies provide overwhelming evidence for overlapping activations between performed actions, imagined actions and MR of body parts ${ }^{25,26,28,29,55}$. Additionally, there is direct behavioral evidence for overlapping cognitive processes activated during real action and MR. Contrary to a widely held view, the nearly monotonous increase of RTs with increasing angular disparity typically observed in MR studies does not sufficiently justify to infer overlapping cognitive processes between MR and real action. The reason is that cognitive systems could in principle simulate continuous, amodal processes (such as a smooth action) by using discrete, conceptual or propositional representations ${ }^{56}$. However, behavioral studies have shown that motor activity and MR produce almost indistinguishable patterns when motor rotation and MR are directly compared ${ }^{57}$, and even interfere with each other when they are aimed in opposite directions ${ }^{58-60}$. These findings strongly support the conclusion that MR not only 'mimics' real manual rotation, but truly shares cortical resources as well as underlying cognitive components.

Regarding the second assumption, our electrophysiological results show that MR of objects and MR of hands are executed in a similar sequence of processing steps. Even though the temporal segmentation of EEG patterns suggests that Map 6 was particularly present for rotated hands, this is merely a relative difference and does not 
exclude presence of the processing steps associated with this map in the other conditions. Indeed, Map 6 is particularly interesting with respect to the possible involvement of motor processes because source analyses of this map not only revealed activations of the right angular gyrus, but also of dorsal primary motor and somatosensory cortex (see Figure 5). These EEG sources were present for both types of stimuli, and may be interpreted as an automatic activation of motor representations during the spatial transformation of mental images. Our findings thus not only complement previous fMRI studies by showing that brain regions necessary for motor execution are recruited during MR, but also show that this activation occurs simultaneously with the right angular gyrus, a brain region that is critical for spatial cognition.

\section{Conclusion}

Our study reveals largely overlapping processing stages and sources of EEG activity when participants perform MR of objects or hands. In agreement with previous studies, we identified an early processing stage differentiating between the two stimulus types, which is compatible with a rapid stimulus classification mechanism. We additionally describe three later processing stages reflecting the spatial transformation associated with MR as well as decision processes related to response programming. Finally, our data show that one of these later stages generates activations of the right angular gyrus and primary motor/somatosensory cortex. These findings indicate that MR of objects and body parts recruits bodily representations and thus entails an emulation of low-level motor processes.

\section{Methods}

\section{Participants}

Twenty-six right-handed healthy participants with normal or corrected-to-normal vision participated to this study. No participant had a history of neurological or psychiatric disorders. Due to high levels of noise in the EEG data, 8 subjects were excluded from the analysis. Data from the remaining 18 participants (11 females) aged 19-39 years $(M=25.33, S D=5.24)$ was used for analysis. The study was approved by the Ethical Commission of the canton of Geneva, and all research was performed in accordance with relevant guidelines/regulations. Participants gave written informed consent before being enrolled.

\section{Stimuli and procedure}

Hands and chairs were used as rotation stimuli. MR with hands is hypothesized to rely on a Ml strategy and therefore is commonly used to assess $\mathrm{Ml}$ ability ${ }^{61}$. Since this task requires participants to identify a hand shown in an upright or rotated position, we chose chairs for the control condition because they can easily be seen as oriented to the left or right when shown in their canonical (upright) position. This ensured that participants produced the same kind of response for both types of stimuli (saying "left" for the left hand/leftward oriented chair, and "right" for the right hand/rightward oriented chair). Stimuli were black and white photographs of three right hands (two feminine, one masculine) seen palm-down, and three different types of chairs. For each stimulus type a mirror image was produced and for each left/right stimulus a rotated version at six different angular disparities $\left(0^{\circ}, 60^{\circ}, 120^{\circ}, 180^{\circ}, 240^{\circ}, 300^{\circ}\right)$ was created. Stimuli were $21.4^{\circ}$ along their longer axis $\left(16.1^{\circ}\right.$ along their short axis) and were shown in grey-scale on black background. 
Participants were tested in a sound-proof EEG chamber. Stimuli were presented centrally on a computer screen located $57 \mathrm{~cm}$ in front of the participants using E-prime (E-studio) software, while EEG recordings were continuously registered. In order to favor $\mathrm{MI}$ in the hand rotation task we did not measure manual reaction times (which might interfere with MI involving the hands), but oral responses. Participant's hands were placed on their knees and covered with a cloth to prevent subjects from looking at their hands while performing the task. The oral response was recorded with a microphone placed about $10 \mathrm{~cm}$ in front of the participants' lips.

Before running the experiment, participants practiced 25 trials to get familiarized with the task. Each trial began with the presentation of a central fixation cross for $1000 \mathrm{~ms}$ to indicate the upcoming stimulus. Participants were asked to maintain fixation on the cross and to suppress eye blinks during the trials. The rotation stimulus was then presented for $2000 \mathrm{~ms}$, and participants gave their oral response. There were nine trials for each rotational disparity $\left(0^{\circ}, 60^{\circ}, 120^{\circ}, 180^{\circ}, 240^{\circ}, 300^{\circ}\right)$ and stimulus orientation (left/right) shown in randomized order, resulting in 108 trials per block. Hands and chairs were presented in distinct blocks, with five blocks of each stimulus type. Half of the participants started with hands, the other half with chairs. Participants were told that only the responses 'right' and 'left' were allowed and were instructed to answer as quickly and accurately as possible. Each block was followed by a short break, and a larger break ( five minutes) was allowed before starting the second half of the experiment.

\section{Behavioral analyses}

Oral responses were registered individually in files of $2000 \mathrm{~ms}$ starting at target onset. RTs and responses were analyzed offline with a custom-made script in MATLAB, which allowed measuring the onset of the response individually with a semi-automatized procedure. Error rates and RTs (correct trials only) were analyzed with a repeated-measures analyses of variance (rANOVA) with the within-subjects factors Stimulus type (chair/hand) and Angular disparity $\left(0^{\circ}, 60^{\circ}, 120^{\circ}, 180^{\circ}\right)$. Where appropriate the Greenhouse-Geisser correction was applied to correct for sphericity violations. Post-hoc Bonferroni-corrected paired t-tests were performed on the highestlevel significant interactions.

\section{EEG acquisition and pre-processing}

Continuous high-density electroencephalography (EEG) recordings were acquired using a 156-channel Brainvision actiCHamp system (Brain Products $\mathrm{GmbH}$, Germany) at sampling frequency of $1000 \mathrm{~Hz}$. During EEG installation, electrode impedance was kept under $20 \mathrm{k} \Omega$ at each electrode.

EEG data pre-processing was performed in Cartool software (https://sites.google.com/site/fbmlab/cartool; ${ }^{62}$. The EEG was filtered off-line between 1 and $30 \mathrm{~Hz}$. Epochs from $100 \mathrm{~ms}$ pre-stimulus to $600 \mathrm{~ms}$ post-stimulus were considered for the ERPs analysis. EEG-epochs were visually inspected to exclude epochs characterized by the presence of artifacts (eye blinks or movements, muscle contractions, sweating or environmental factors) were excluded. Artifact-free epochs were averaged per condition for each subject. Before averaging across subjects, bad channels from each participant carrying repetitive artifacts during prolonged periods were interpolated from neighboring electrodes using 3D spline interpolation ( $<10 \%$ interpolated electrodes) ${ }^{63}$.

An rANOVA with the factors Stimulus type and Angular disparity showed no statistical difference between the number of epochs accepted for each Stimulus type $\left(F_{1,17}=0.133, p=0.719, \eta^{2}=0.008\right)$, but revealed a significant effect of Angular disparity $\left(F_{2.73,46.56}=7.29, p<0.01, \eta^{2}=0.3\right)$. Post-hoc tests showed that this difference was due to a significantly lower number of correct responses for stimuli rotated by $300^{\circ}$ than all other disparities (all $p<0.01$ ). For this reason, EEG data were only analyzed for angular disparities $0^{\circ}$ and $180^{\circ}$, as has been done 
in several previous studies. This reduced EEG analyses to the following four experimental conditions: Chair $0^{\circ}$, Hand $0^{\circ}$, Chair $180^{\circ}$, Hand $180^{\circ}$.

\section{Global amplitude analysis}

To determine whether Stimulus type, Angular disparity and/or the interaction of both factors induced significant ERP amplitude differences across the whole electrode set, a waveform non-parametric repeated-measures ANOVA was performed as exploratory analysis with the Statistical Toolbox for Electrical Neuroimaging (STEN; http://doi.org/10.5281/zenodo.1164038) ${ }^{64}$. Family-wise error (FWE) was controlled through bootstrapping ( $\mathrm{N}=$ 1000 permutations) for a significance level $\alpha=0.05$. In addition, we applied constraining criteria regarding electrode cluster-size (only clusters consisting of minimum seven neighboring electrodes were retained) and temporal clustering (only differences that were contiguous across at least 20 time-frames were accepted).

\section{ERP segmentation and statistical assessment}

To identify periods of stable scalp voltage configurations (scalp EEG topographies or 'microstates') and capture the differences between the experimental conditions, ERP cluster segmentation was performed ${ }^{62,65,66}$. Following visual inspection of the global field power (GFP) and dissimilarity curves of averaged ERPs for the four conditions (Chairs/Hands; $0^{\circ} / 180^{\circ}$ ) we restricted the segmentation to the period between $90 \mathrm{~ms}$ and $500 \mathrm{~ms}$ post-onset. This also allowed us to focus on electrophysiological signals that were relatively uncontaminated by processes related to the verbal response (the fastest responses being $>800 \mathrm{~ms}$, see results). For the segmentation, a kmeans clustering algorithm with 300 randomizations was used, whereby the minimal duration of maps was set to $30 \mathrm{~ms}$ and cluster solutions ranging from 1 to 30 clusters were examined ${ }^{62,65,66}$. The optimal number of topographic clusters (henceforth termed 'maps') that best described the group-averaged ERPs of the four conditions was determined with a meta-criterion which integrated the results of seven independent optimization criteria ${ }^{67}$.

To assess statistical differences of the obtained cluster maps between the different ERPs conditions, statistical analysis was performed using a topographic non-parametric (rank-based) randomization procedure implemented in RAGU software ${ }^{68}$. This approach evaluates for each map the statistical difference between conditions by permuting for a defined number of times the data of the grand averaged ERPs. The permutation-based analysis compares the presence of cluster maps to the distribution obtained under the null hypothesis. This method of controlling FWE has higher statistical power than if it were based on individual subjects' ERPs, known to have high variance ${ }^{68}$. We applied 1000 permutations and set a threshold of significance to $p=0.05$. The difference between maps is given in terms of six parameters: first (onset) and last (offset) assignment of the ERP to the map, duration (mean of the time points assigned to a given map), area under the curve (AUC; defined as the sum of GFP during all time points assigned to the map), center of gravity (i.e., the time-point associated with the center of the GFP area) and mean GFP (the average strength of activation across all map time points) ${ }^{69}$.

\section{EEG source localization}

In order to estimate the neural current densities underlying EEG scalp topographies obtained with the segmentation, we computed an inverse solution matrix using standardized low resolution electromagnetic 
tomography SLORETA; ${ }^{70}$, which constrains the inverse solution by minimizing the second-order spatial derivative of the current source distribution to maximize spatial coherence. We used as head model the Montreal Neurological Institute (MNI) template (including the cerebellum) and applied the Locally Spherical Model with Anatomical Constraints (LSMAC) which respects local differences of skull thickness corrects for global resistivity value ${ }^{71}$.We defined a grid consisting of 6000 solution points distributed equally in the gray matter. Considering the obtained transformation (inverse) matrix for each subject, we then inverted in the source space (i.e., template brain) each epoch corresponding to the four experimental conditions (Chairs/Hands, $0^{\circ} / 180^{\circ}$ ). We then averaged for each subject the inverted epochs belonging to the same condition, and finally computed a grand average across subjects. This approach normalizes the power of the current density across all solution points and corrects for background noise, resulting in an increased Signal-to Noise ratio and improved detection of those solution points that exhibit maximal activation ${ }^{67}$.

Specific time windows of group-averaged ERPs in the inverse space were selected to visualize the activation of the neuronal sources based on the results of the statistic of cluster maps between conditions. To better characterize the neuronal sources, in such windows, volumes representing the activation above the $95^{\text {th }}$ percentile were extracted in $30 \mathrm{~ms}$ steps, for each condition.

Data availability: The datasets generated during this study are available from the University of Geneva Yareta repository by using these links: https://doi.org/10.26037/yareta:uoksutig4zaghb5i3s26dv5iqa (behavioral data) and https://doi.org/10.26037/yareta:qkhx74c27vbmbh7qfbfog4ja4y (EEG data). 


\section{References}

Thelen, E. Grounded in the world: developmental origins of the embodied mind. Infancy 1, 3-28 (2000).

Barsalou, L. W. Grounded cognition. Annu Rev Psychol 59, 617-645, doi:10.1146/annurev.psych.59.103006.093639 (2008).

Dehaene, S. et al. How learning to read changes the cortical networks for vision and language. Science 330, 13591364, doi:10.1126/science.1194140 (2010).

Anderson, M. L. Neural reuse: A fundamental organizational principle of the brain. Behav. Brain Sci. 33, 245-313 (2010).

Wilson, M. Six views of embodied cognition. Psychon Bull Rev 9, 625-636 (2002).

Caramazza, A., Anzellotti, S., Strnad, L. \& Lingnau, A. Embodied cognition and mirror neurons: a critical assessment. Annu Rev Neurosci 37, 1-15, doi:10.1146/annurev-neuro-071013-013950 (2014).

Hauk, O., Johnsrude, I. \& Pulvermüller, F. Somatotopic representation of action words in human motor and premotor cortex. Neuron 41, 301-307 (2004).

Goldinger, S. D., Papesh, M. H., Barnhart, A. S., Hansen, W. A. \& Hout, M. C. The poverty of embodied cognition. Psychon Bull Rev 23, 959-978, doi:10.3758/s13423-015-0860-1 (2016).

Shepard, R. N. \& Metzler, J. Mental rotation of three-dimensional objects. Science 171, 701-703 (1971).

Searle, J. A. \& Hamm, J. P. Mental rotation: an examination of assumptions. Wiley Interdiscip Rev Cogn Sci 8, doi:10.1002/wcs.1443 (2017).

Jansen, P. \& Heil, M. The relation between motor development and mental rotation ability in 5- to 6-year old children. European Journal of Developmental Science 4, 66-74 (2010).

Cona, G. \& Scarpazza, C. Where is the "where" in the brain? A meta-analysis of neuroimaging studies on spatial cognition. Hum Brain Mapp 40, 1867-1886, doi:10.1002/hbm.24496 (2019).

Vingerhoets, G. Contribution of the posterior parietal cortex in reaching, grasping, and using objects and tools. Frontiers in psychology 5, 151, doi:10.3389/fpsyg.2014.00151 (2014). pointing. Neuroscientist 16, 388-407, doi:10.1177/1073858410375468 (2010). Trends Cogn. Sci. 21, 589-599, doi:10.1016/j.tics.2017.05.002 (2017). Likelihood Estimation Meta-Analysis. Front Hum Neurosci 9, 693, doi:10.3389/fnhum.2015.00693 (2015).

Jola, C. \& Mast, F. W. Mental object rotation and egocentric body transformation: two dissociable processes? Spatial Cognition and Computation 5, 217-237 (2005). 
Coslett, H. B., Medina, J., Kliot, D. \& Burkey, A. R. Mental motor imagery indexes pain: the hand laterality task. Eur J Pain 14, 1007-1013, doi:10.1016/j.ejpain.2010.04.001 (2010). de Lange, F. P., Helmich, R. C. \& Toni, I. Posture influences motor imagery: an fMRI study. Neurolmage 33, 609617, doi:10.1016/j.neuroimage.2006.07.017 (2006). Parsons, L. M. Imagined spatial transformations of one's hands and feet. Cogn. Psychol. 19, 178-241 (1987). Sekiyama, K. Kinesthetic aspects of mental representations in the identification of left and right hands. Percept. Psychophys. 32, 89-95 (1982).

Cohen, M. S. et al. Changes in cortical activity during mental rotation. A mapping study using functional MRI. Brain 119 ( Pt 1), 89-100, doi:10.1093/brain/119.1.89 (1996).

Kosslyn, S. M., DiGirolamo, G. J., Thompson, W. L. \& Alpert, N. M. Mental rotation of objects versus hands: neural mechanisms revealed by positron emission tomography. Psychophysiology 35, 151-161 (1998).

Jordan, K., Wustenberg, T., Heinze, H. J., Peters, M. \& Jancke, L. Women and men exhibit different cortical activation patterns during mental rotation tasks. Neuropsychologia 40, 2397-2408, doi:10.1016/s00283932(02)00076-3 (2002).

Wraga, M., Shephard, J. M., Church, J. A., Inati, S. \& Kosslyn, S. M. Imagined rotations of self versus objects: an fMRI study. Neuropsychologia 43, 1351-1361, doi:10.1016/j.neuropsychologia.2004.11.028 (2005).

Milivojevic, B., Hamm, J. P. \& Corballis, M. C. Functional neuroanatomy of mental rotation. J. Cogn. Neurosci. 21, 945-959, doi:10.1162/jocn.2009.21085 (2009).

Lamm, C., Windischberger, C., Moser, E. \& Bauer, H. The functional role of dorso-lateral premotor cortex during mental rotation: an event-related fMRI study separating cognitive processing steps using a novel task paradigm. Neurolmage 36, 1374-1386, doi:10.1016/j.neuroimage.2007.04.012 (2007).

Richter, W. et al. Motor area activity during mental rotation studied by time-resolved single-trial fMRI. J. Cogn. Neurosci. 12, 310-320, doi:10.1162/089892900562129 (2000).

Zacks, J. M. Neuroimaging studies of mental rotation: a meta-analysis and review. J. Cogn. Neurosci. 20, 1-19, doi:10.1162/jocn.2008.20013 (2008).

Vingerhoets, G., de Lange, F. P., Vandemaele, P., Deblaere, K. \& Achten, E. Motor imagery in mental rotation: an fMRI study. Neurolmage 17, 1623-1633 (2002).

Pegna, A. J. et al. Unraveling the cerebral dynamics of mental imagery. Hum Brain Mapp 5, 410-421, doi:10.1002/(SICI)1097-0193(1997)5:6 (1997).

Peronnet, F. \& Farah, M. J. Mental rotation: an event-related potential study with a validated mental rotation task. Brain. Cogn. 9, 279-288, doi:10.1016/0278-2626(89)90037-7 (1989). 
Thayer, Z. C. \& Johnson, B. W. Cerebral processes during visuo-motor imagery of hands. Psychophysiology 43, 401-412, doi:10.1111/j.1469-8986.2006.00404.x (2006).

Jansen, P., Render, A., Scheer, C. \& Siebertz, M. Mental rotation with abstract and embodied objects as stimuli: evidence from event-related potential (ERP). Exp. Brain Res. 238, 525-535, doi:10.1007/s00221-020-05734-w (2020).

Dalecki, M., Hoffmann, U. \& Bock, O. Mental rotation of letters, body parts and complex scenes: separate or common mechanisms? Hum Mov Sci 31, 1151-1160, doi:10.1016/j.humov.2011.12.001 (2012).

Reed, C. L., Stone, V. E., Bozova, S. \& Tanaka, J. The body-inversion effect. Psychol Sci 14, 302-308, doi:10.1111/1467-9280.14431 (2003).

Pegna, A. J., Khateb, A., Michel, C. M. \& Landis, T. Visual recognition of faces, objects, and words using degraded stimuli: where and when it occurs. Hum Brain Mapp 22, 300-311, doi:10.1002/hbm.20039 (2004).

Johnson, J. S. \& Olshausen, B. A. Timecourse of neural signatures of object recognition. J Vis 3, 499-512, doi:10.1167/3.7.4 (2003).

Ptak, R., Lazeyras, F., Di Pietro, M., Schnider, A. \& Simon, S. R. Visual object agnosia is associated with a breakdown of object-selective responses in the lateral occipital cortex. Neuropsychologia 60, 10-20 (2014).

Feinberg, T. E., Schindler, R. J., Ochoa, E., Kwan, P. C. \& Farah, M. J. Associative visual agnosia and alexia without prosopagnosia. Cortex 30, 395-411 (1994).

Downing, P. E., Chan, A. W., Peelen, M. V., Dodds, C. M. \& Kanwisher, N. Domain specificity in visual cortex. Cereb. Cortex 16, 1453-1461, doi:10.1093/cercor/bhj086 (2006).

Taylor, J. C. \& Downing, P. E. Division of labor between lateral and ventral extrastriate representations of faces, bodies, and objects. J. Cogn. Neurosci. 23, 4122-4137, doi:10.1162/jocn_a_00091 (2011).

Xue, J. et al. Uncovering the cognitive processes underlying mental rotation: an eye-movement study. Sci Rep 7, 10076, doi:10.1038/s41598-017-10683-6 (2017).

Just, M. A. \& Carpenter, P. A. Eye fixations and cognitive processes. Cogn. Psychol. 8, 441-480 (1976). (2009).

Sack, A. T. et al. Imaging the brain activity changes underlying impaired visuospatial judgments: simultaneous FMRI, TMS, and behavioral studies. Cereb. Cortex 17, 2841-2852, doi:10.1093/cercor/bhm013 (2007). dichotomy. Neuropsychologia 47, 1434-1448, doi:10.1016/j.neuropsychologia.2008.11.033 (2009). doi:10.1177/1073858412440596 (2013). 
Schendan, H. E. \& Maher, S. M. Object knowledge during entry-level categorization is activated and modified by implicit memory after 200 ms. Neurolmage 44, 1423-1438, doi:10.1016/j.neuroimage.2008.09.061 (2009). 31-47, doi:10.1016/j.neunet.2015.09.010 (2015). (Basel) 18, doi:10.3390/s18092920 (2018).

Overney, L. S., Michel, C. M., Harris, I. M. \& Pegna, A. J. Cerebral processes in mental transformations of body parts: recognition prior to rotation. Brain Res Cogn Brain Res 25, 722-734, doi:10.1016/j.cogbrainres.2005.09.024 (2005).

Hardwick, R. M., Caspers, S., Eickhoff, S. B. \& Swinnen, S. P. Neural correlates of action: Comparing metaanalyses of imagery, observation, and execution. Neurosci. Biobehav. Rev. 94, 31-44, doi:10.1016/j.neubiorev.2018.08.003 (2018).

Pylyshyn, Z. W. What the mind's eye tells the mind's brain: A critique of mental imagery. Psychol. Bull. 80, 1-24 (1973).

Gardony, A. L., Taylor, H. A. \& Brunye, T. T. What does physical rotation reveal about mental rotation? Psychol Sci 25, 605-612, doi:10.1177/0956797613503174 (2014).

Wexler, M., Kosslyn, S. M. \& Berthoz, A. Motor processes in mental rotation. Cognition 68, 77-94 (1998).

Wohlschlager, A. Mental object rotation and the planning of hand movements. Percept. Psychophys. 63, 709-718 (2001).

Wohlschlager, A. \& Wohlschlager, A. Mental and manual rotation. J Exp Psychol Hum Percept Perform 24, 397412 (1998).

Boonstra, A. M. et al. Using the Hand Laterality Judgement Task to assess motor imagery: a study of practice effects in repeated measurements. International Journal of Rehabilitation Research 35, 278-280 (2012).

Brunet, D., Murray, M. M. \& Michel, C. M. Spatiotemporal analysis of multichannel EEG: CARTOOL. Computational intelligence and neuroscience 2011, 813870, doi:10.1155/2011/813870 (2011).

Perrin, F., Pernier, J., Bertrand, O., Giard, M. H. \& Echallier, J. F. Mapping of scalp potentials by surface spline interpolation. Electroencephalography and Clinical Neurophysiology 66, 75-81 (1987).

Knebel, J. F. \& Notter, M. P. STEN 1.0: Statistical Toolbox for Electrical Neuroimaging (Version 1.0). Zenodo, doi:10.5281/zenodo.1167723 (2012).

Murray, M. M., Brunet, D. \& Michel, C. M. Topographic ERP analyses: a step-by-step tutorial review. Brain Topography 20, 249-264 (2008).

Pasqual-Marqui, R. D., Michel, C. M. \& Lehmann, D. Segmentation of brain electrical activity into microstates: model estimation and validation. IEEE Transactions on Biomedical Engineering 42, 658-665 (1995). 
67 Brechet, L. et al. Capturing the spatiotemporal dynamics of self-generated, task-initiated thoughts with EEG and fMRI. Neurolmage 194, 82-92, doi:10.1016/j.neuroimage.2019.03.029 (2019).

68 Koenig, T., Stein, M., Grieder, M. \& Kottlow, M. A tutorial on data-driven methods for statistically assessing ERP topographies. Brain Topogr 27, 72-83, doi:10.1007/s10548-013-0310-1 (2014).

69 Habermann, M., Weusmann, D., Stein, M. \& Koenig, T. A Student's Guide to Randomization Statistics for Multichannel Event-Related Potentials Using Ragu. Front Neurosci 12, 355, doi:10.3389/fnins.2018.00355 (2018).

70 Pascual-Marqui, R. D. Standardized low-resolution brain electromagnetic tomography (sLORETA): technical details. Methods Find Exp Clin Pharmacol 24 Suppl D, 5-12 (2002).

71 Michel, C. M. \& Brunet, D. EEG Source Imaging: A Practical Review of the Analysis Steps. Front Neurol 10, 325, doi:10.3389/fneur.2019.00325 (2019). 
Acknowledgement: Study supported by the Swiss National Science Foundation (grant 32003B-184702).

Author contributions: M.M.G. conceived the design, performed the study, analyzed the results and wrote the main manuscript text. A.D. supervised the study. G.R.I. analyzed the results. R.P. conceived the design and wrote the main manuscript text. All authors reviewed and corrected the manuscript. 
Table 1. Summary of statistical effects characterizing the maps 1, 6, 7 and $8\left({ }^{*}: p<.05 ;{ }^{* *}: p<.01 ;{ }^{* * *}: p<\right.$ .001; AUC: area under the curve; GFP: global field power).

\begin{tabular}{|c|c|c|c|c|}
\hline & Measure & Stimulus type & $\begin{array}{c}\text { Model factor } \\
\text { Angular disparity }\end{array}$ & Interaction \\
\hline Map 1 & $\begin{array}{l}\text { Onset } \\
\text { Offset } \\
\text { Duration } \\
\text { AUC } \\
\text { Center of gravity } \\
\text { Mean GFP }\end{array}$ & $* *$ & & \\
\hline Map 6 & $\begin{array}{l}\text { Onset } \\
\text { Offset } \\
\text { Duration } \\
\text { AUC } \\
\text { Center of gravity } \\
\text { Mean GFP }\end{array}$ & & & $* * *$ \\
\hline Map 7 & $\begin{array}{l}\text { Onset } \\
\text { Offset } \\
\text { Duration } \\
\text { AUC } \\
\text { Center of gravity } \\
\text { Mean GFP }\end{array}$ & $\begin{array}{l}* \\
*\end{array}$ & $\begin{array}{l}* * \\
* * \\
* * *\end{array}$ & $\begin{array}{l}\text { * } \\
\text { * } \\
\text { * } \\
\text { ** }\end{array}$ \\
\hline Map 8 & $\begin{array}{l}\text { Onset } \\
\text { Offset } \\
\text { Duration } \\
\text { AUC } \\
\text { Center of gravity } \\
\text { Mean GFP }\end{array}$ & $\begin{array}{l}\text { ** } \\
* \\
* \\
* \\
*\end{array}$ & $\begin{array}{l}\text { *** } \\
* * \\
* * * \\
* * *\end{array}$ & $\stackrel{*}{*}$ \\
\hline
\end{tabular}




\section{Figure legends}

Figure 1. a) Examples of stimulus types shown at each of the six angular disparities. b) Vocal reaction times $( \pm$ SEM) for hands and chairs, as a function of angular disparity.

Figure 2. Results of electrode-wise amplitude analysis for a) the stimulus effect, b) the effect of angular disparity and c) an interaction between both factors. The lower panel shows the number of electrodes that reached significance at each time frame.

Figure 3. a) Spatiotemporal organization of seven maps identified with the EEG segmentation procedure. The height of each curve represents Global field power at each time-point. b). Current voltage topographies corresponding to each of the seven maps.

Figure 4. Current sources underlying Map 1 between 120-150 ms, projected on the MNI template brain. a) Chairs and $b$ ) hands. Only voxels with significance level $p<.05$ are shown.

Figure 5. Current sources underlying Map 6 between 330-360 ms. a) Chairs $0^{\circ}$, b) Chairs $180^{\circ}, \mathrm{c}$ ) Hands $0^{\circ}$ and d) Hands $180^{\circ}$. Only voxels with significance level $p<.05$ are shown.

Figure 6. Current sources underlying Map 7 between $390-420 \mathrm{~ms}$. a) Chairs $0^{\circ}$, b) Chairs $180^{\circ}$, c) Hands $0^{\circ}$ and d) Hands $180^{\circ}$. Only voxels with significance level $p<.05$ are shown.

Figure 7. Current sources underlying Map 8 between $450-480 \mathrm{~ms}$. a) Chairs $0^{\circ}$, b) Chairs $180^{\circ}$, c) Hands $0^{\circ}$ and d) Hands $180^{\circ}$. Only voxels with significance level $p<.05$ are shown. 
a)

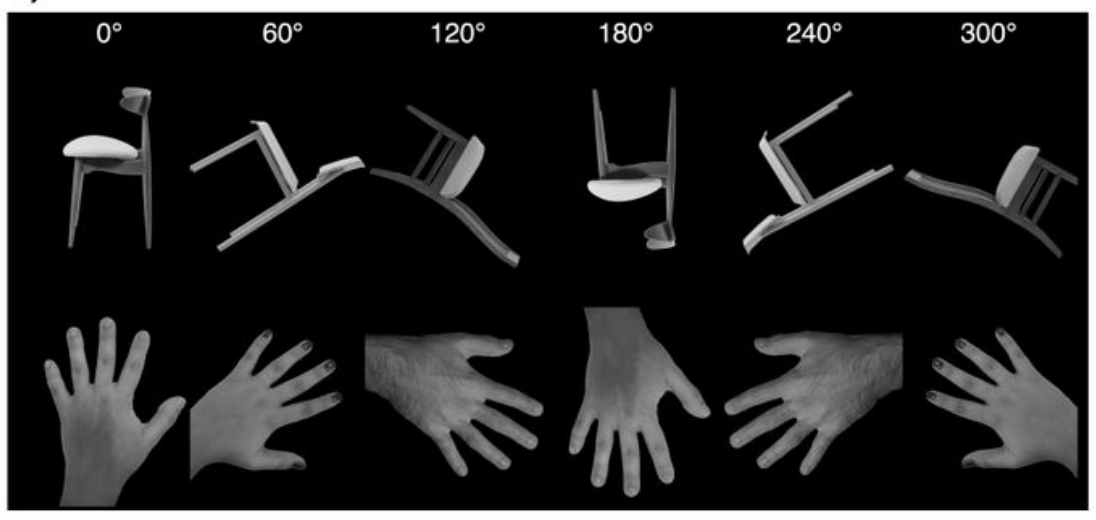

b)

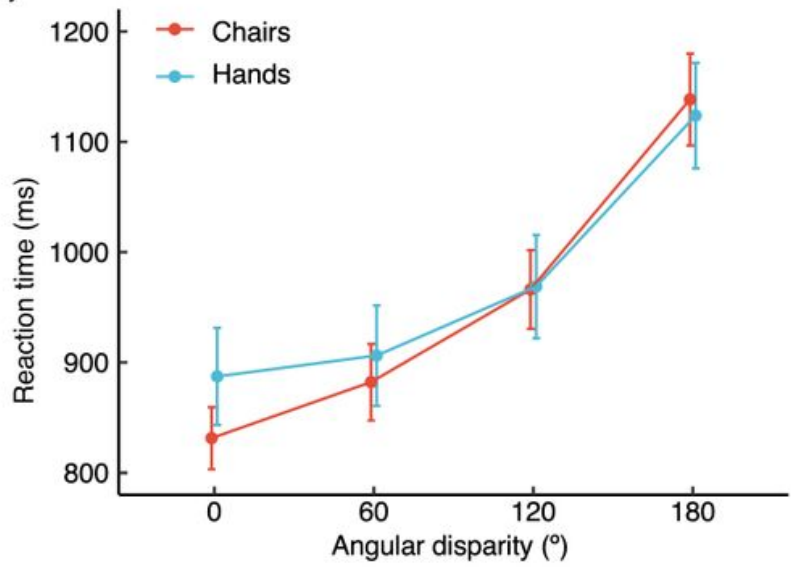

Figure 1

a) Examples of stimulus types shown at each of the six angular disparities. b) Vocal reaction times $( \pm S E M)$ for hands and chairs, as a function of angular disparity.

a)

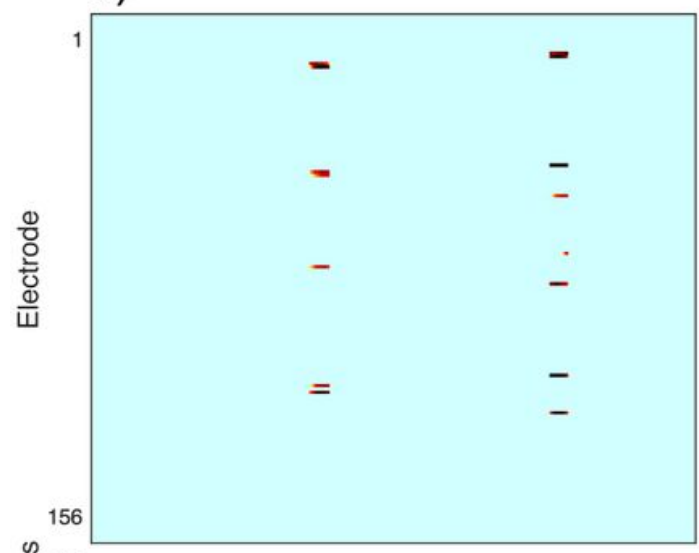

b)

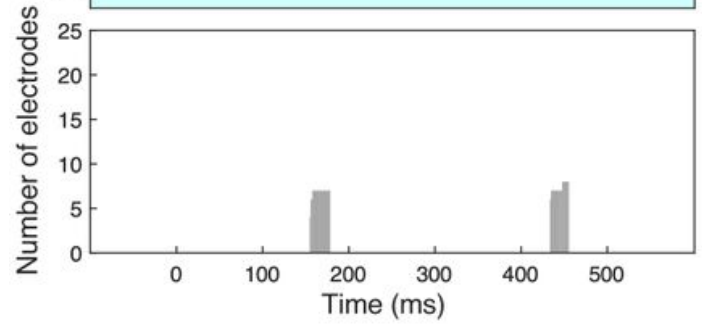

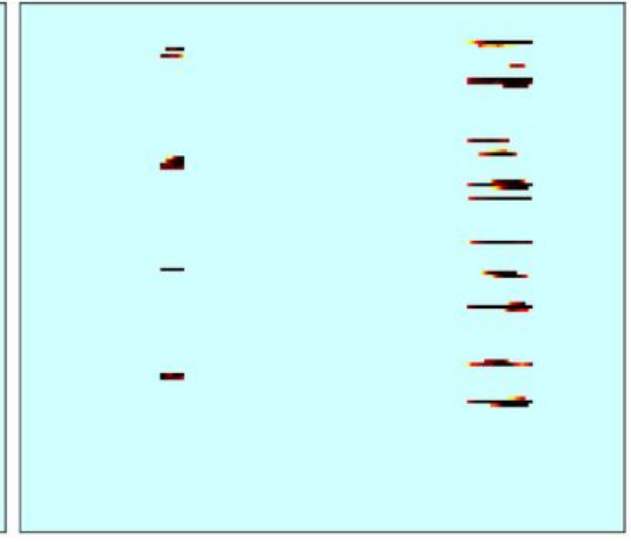

c)

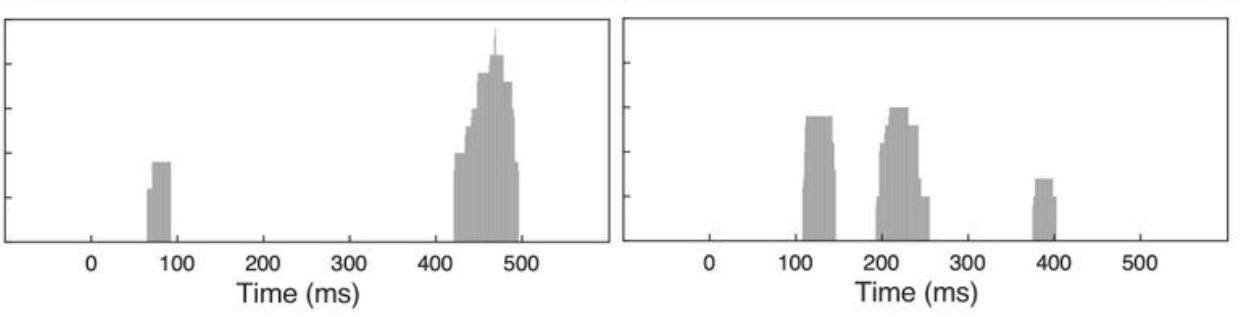

\section{Figure 2}

Results of electrode-wise amplitude analysis for a) the stimulus effect, b) the effect of angular disparity and c) an interaction between both factors. The lower panel shows the number of electrodes that reached significance at each time frame. 


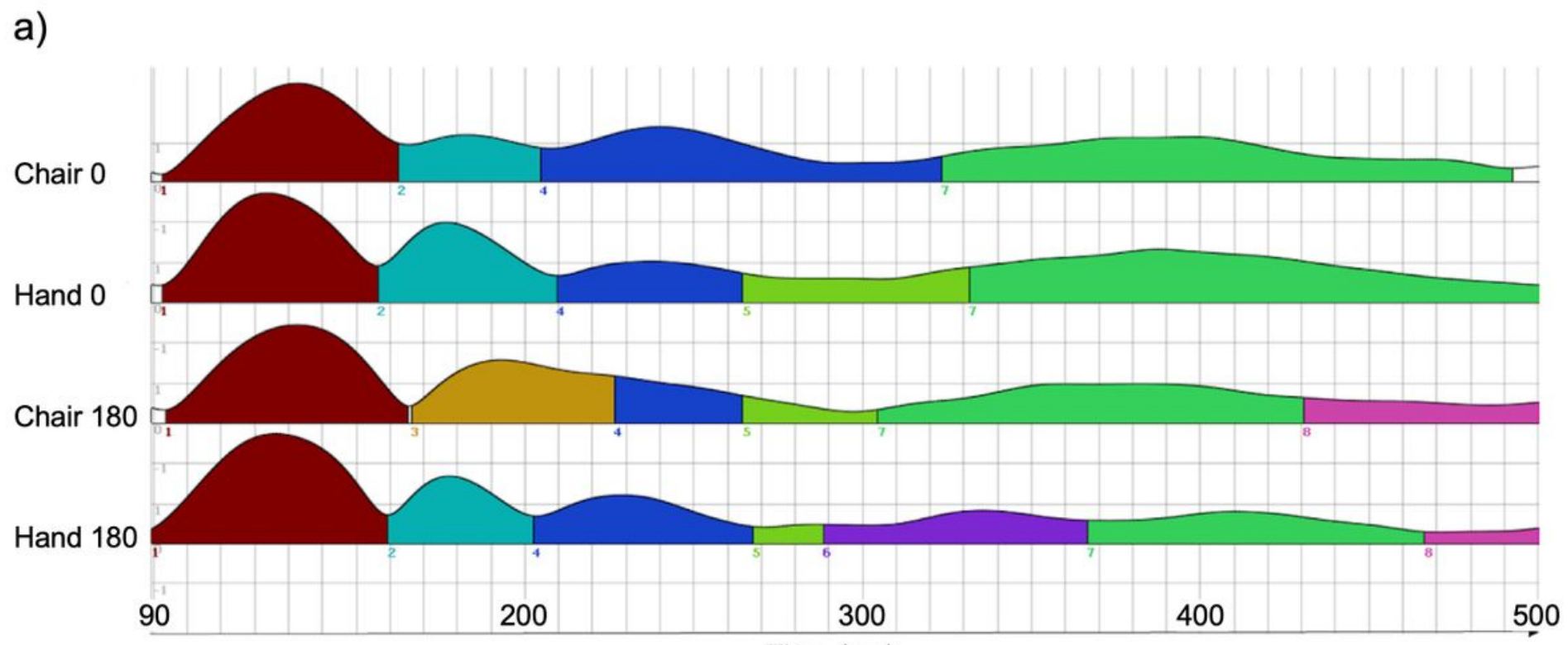

b)

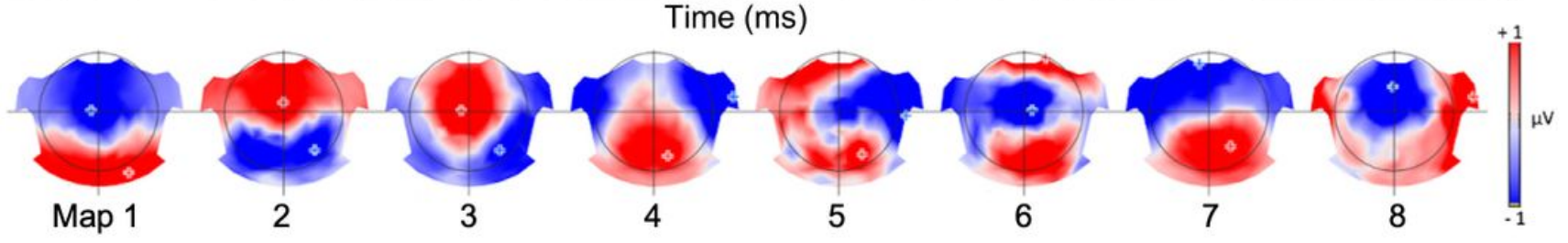

Figure 3

a) Spatiotemporal organization of seven maps identified with the EEG segmentation procedure. The height of each curve represents Global field power at each time-point. b). Current voltage topographies corresponding to each of the seven maps.

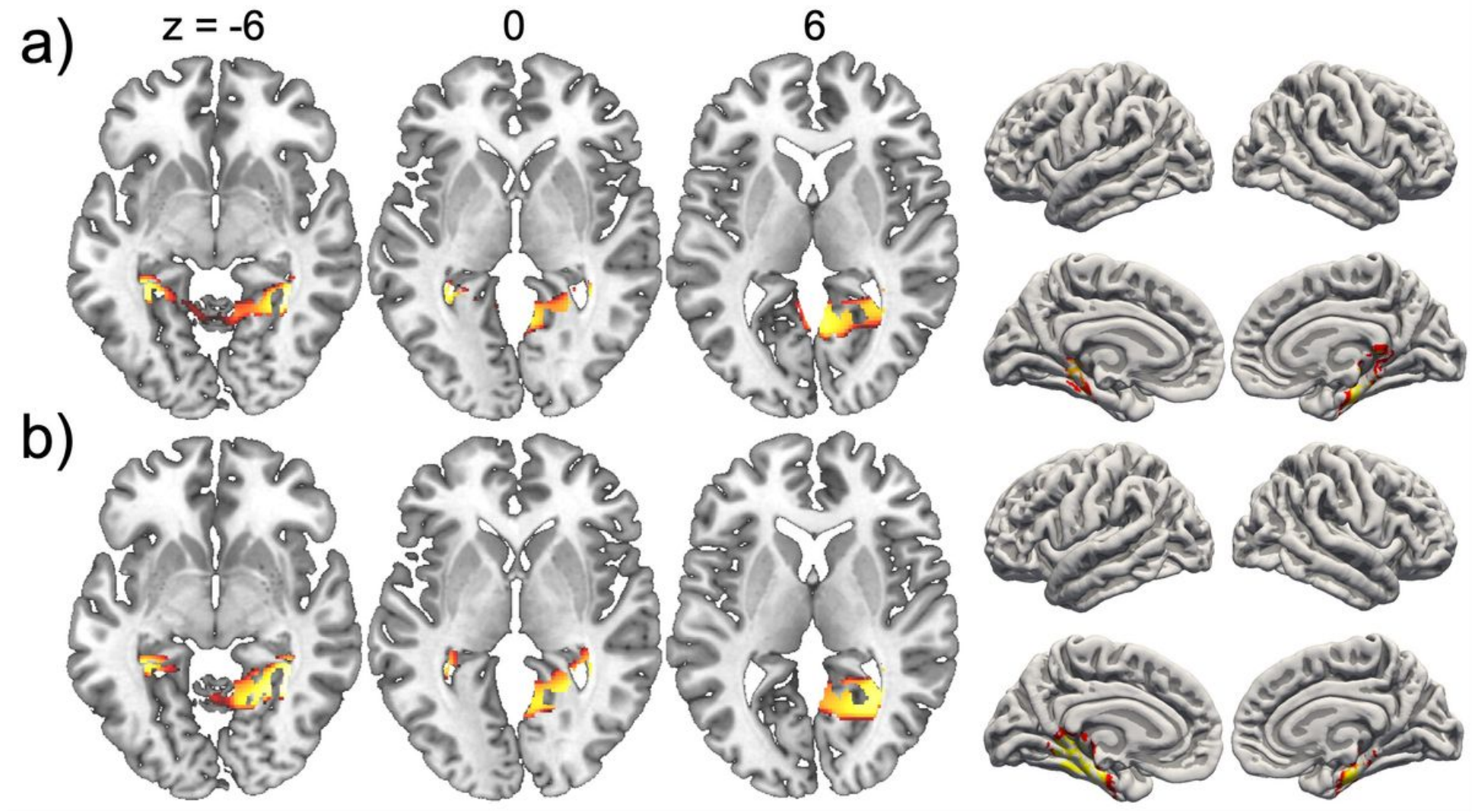




\section{Figure 4}

Current sources underlying Map 1 between 120-150 ms, projected on the MNI template brain. a) Chairs and b) hands. Only voxels with significance level $p<.05$ are shown.

a)
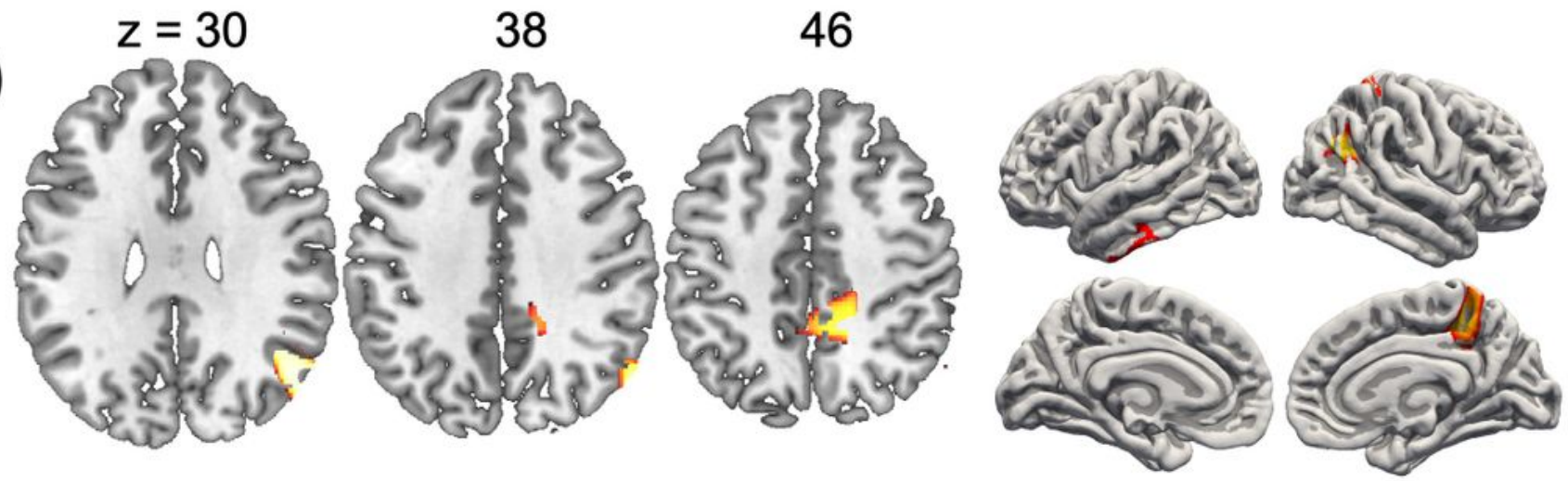

b)
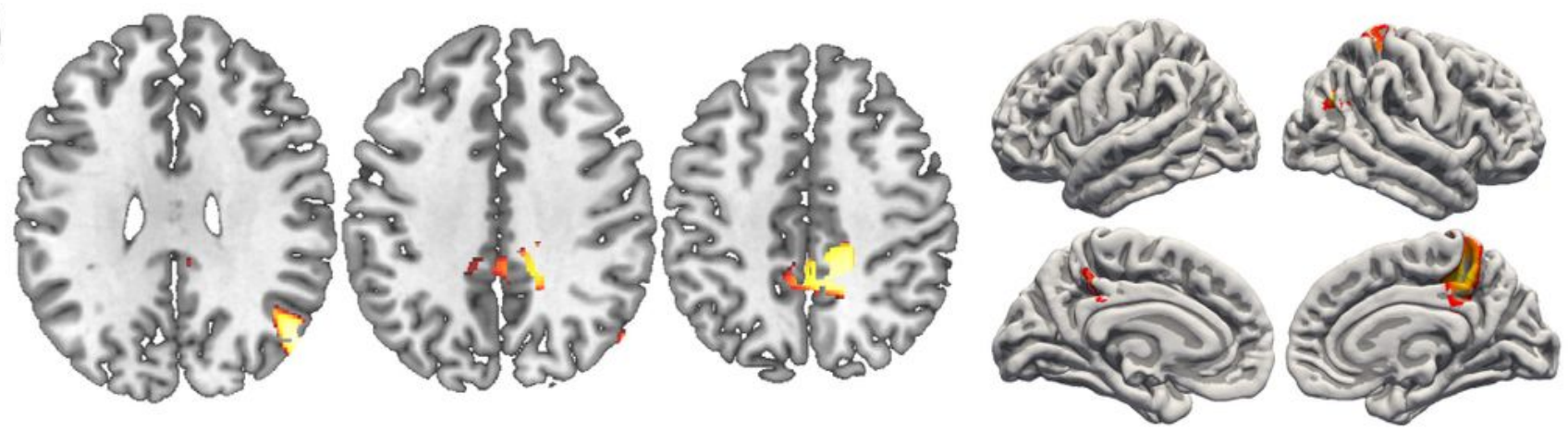

c)
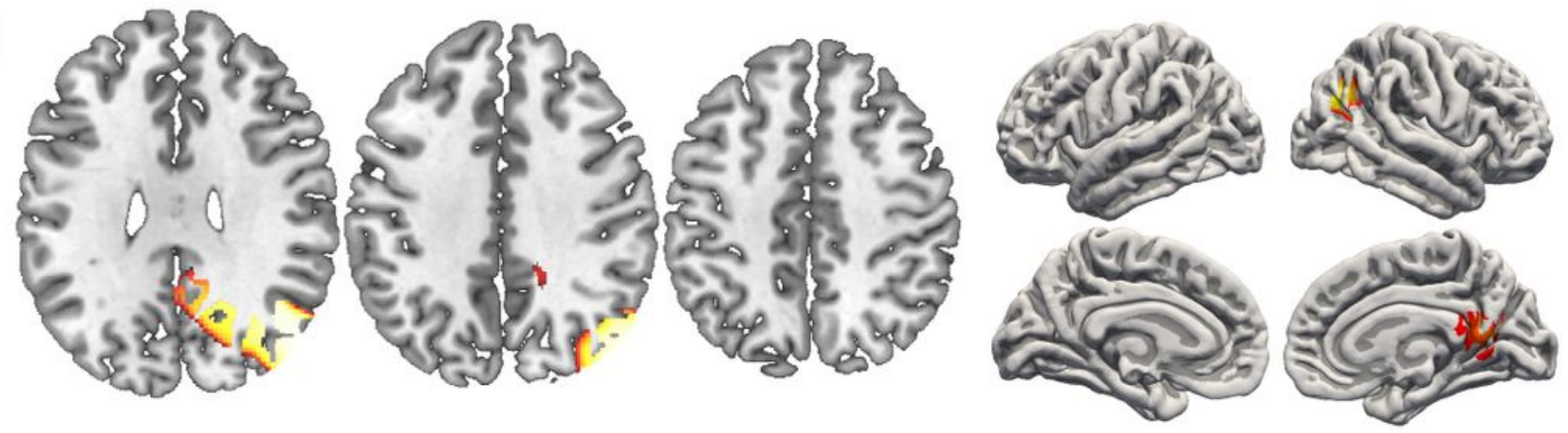

d)
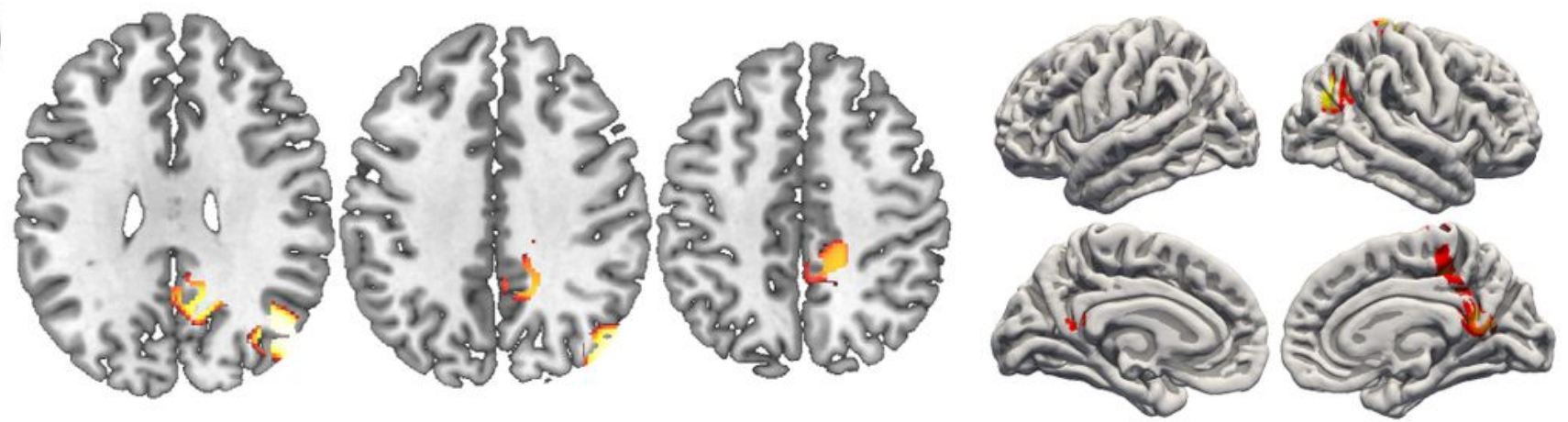

Figure 5 
Current sources underlying Map 6 between 330-360 ms. a) Chairs $0^{\circ}$, b) Chairs $180^{\circ}$, c) Hands $0^{\circ}$ and d) Hands $180^{\circ}$. Only voxels with significance level $p<.05$ are shown.

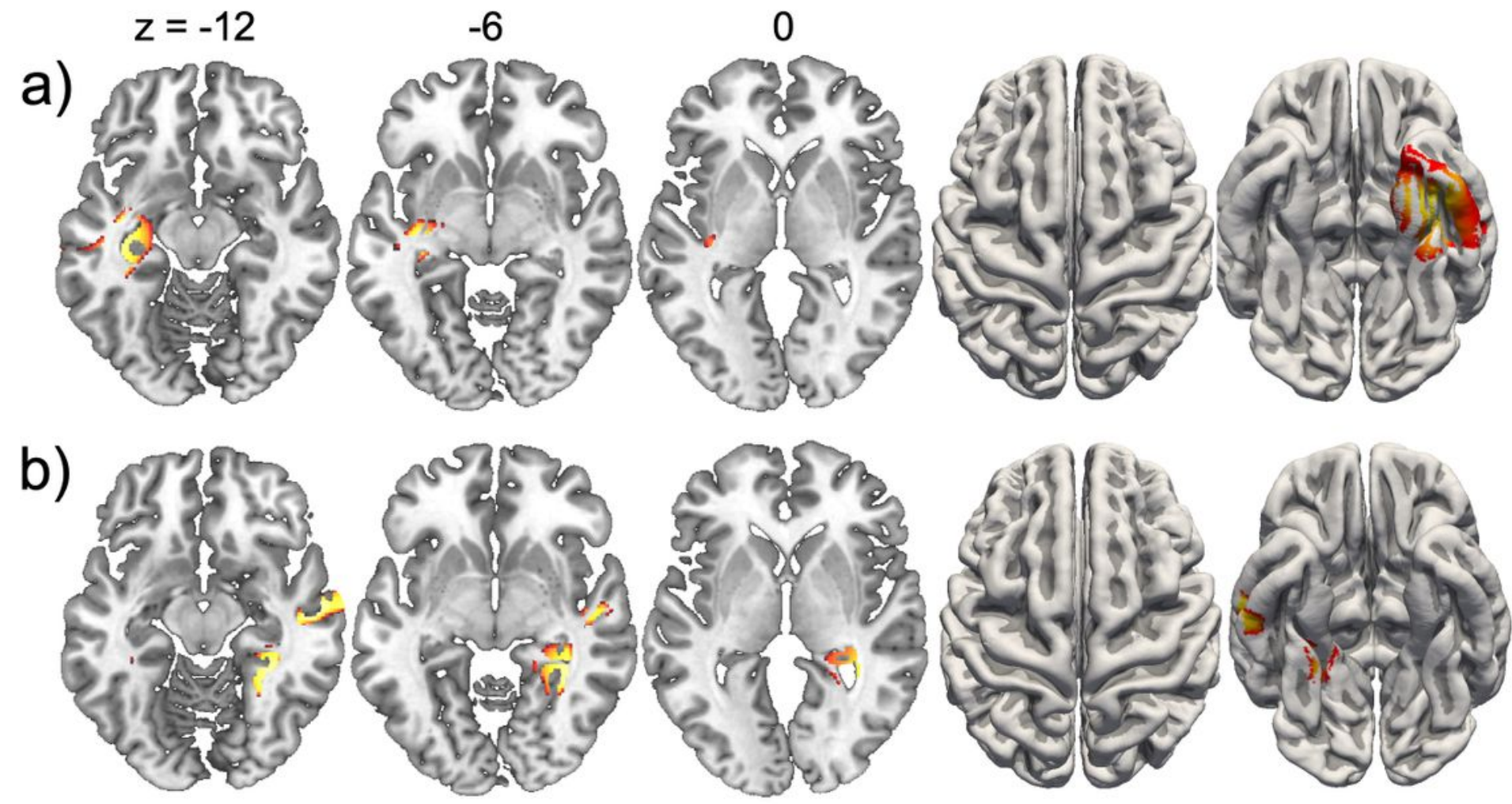

c)
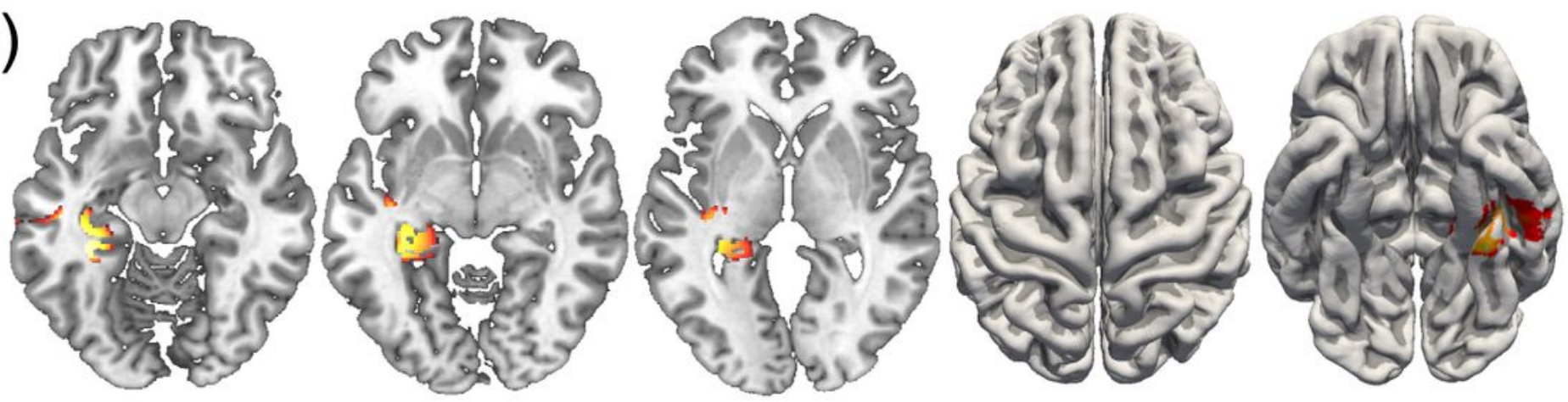

d)
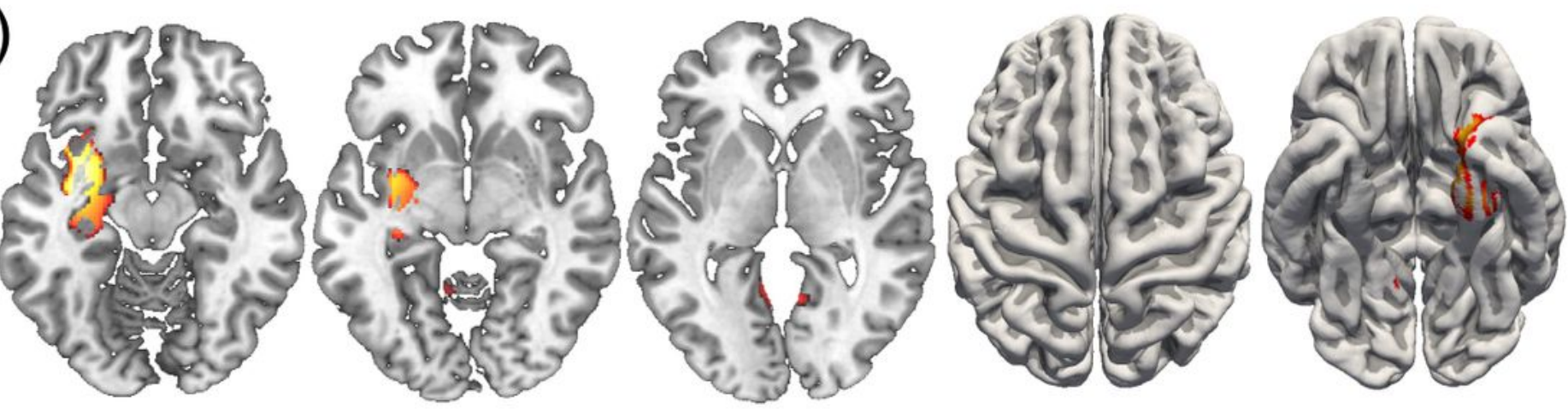

Figure 6

Current sources underlying Map 7 between $390-420 \mathrm{~ms}$. a) Chairs $0^{\circ}$, b) Chairs $180^{\circ}$, c) Hands $0^{\circ}$ and d) Hands $180^{\circ}$. Only voxels with significance level $p<.05$ are shown. 


$$
z=-10 \quad 20 \quad 50
$$

a) nisgen
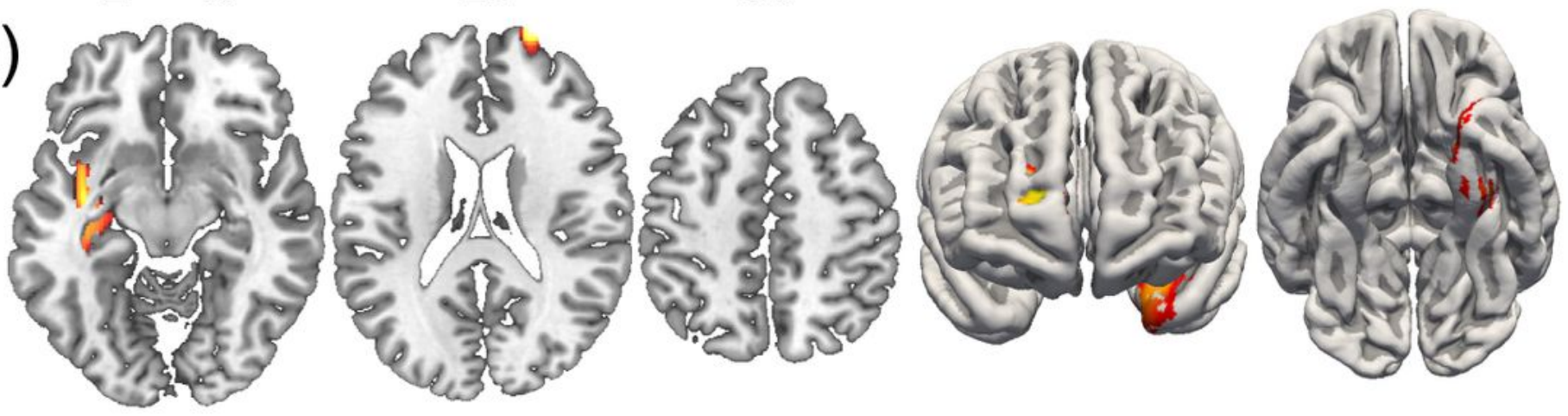

b)
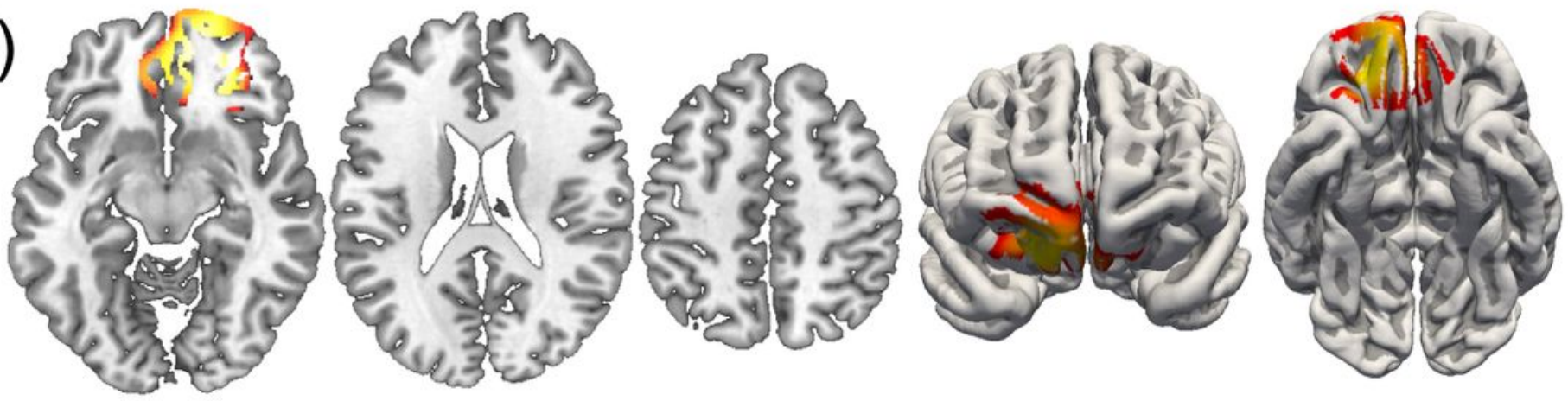

c)
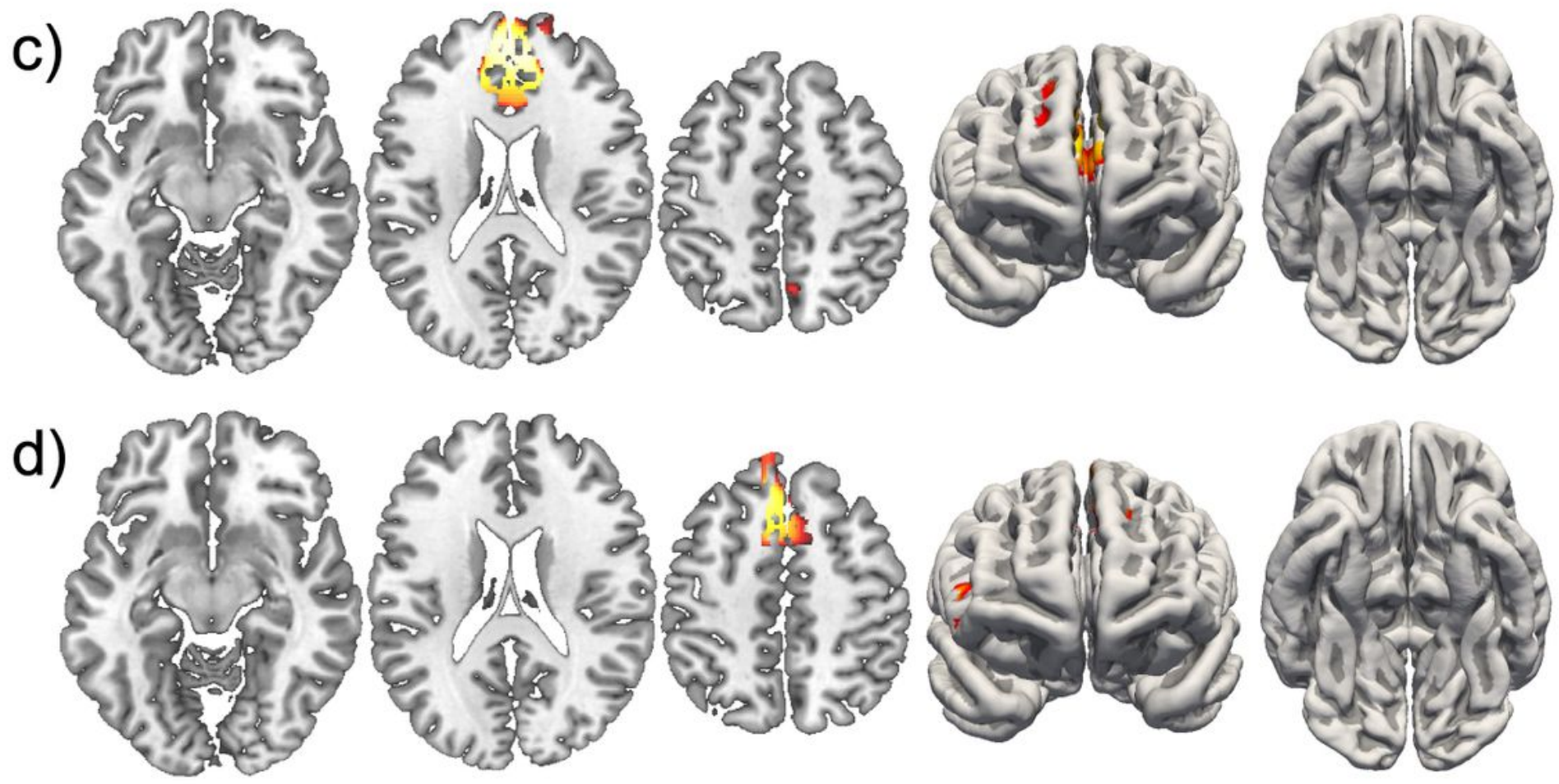

\section{Figure 7}

Current sources underlying Map 8 between 450-480 ms. a) Chairs $0^{\circ}$, b) Chairs $180^{\circ}$, c) Hands $0^{\circ}$ and d) Hands $180^{\circ}$. Only voxels with significance level $p<.05$ are shown. 